International Journal of Pure and Applied Mathematics

Volume 89 No. $1 \quad 2013$, 29-33

ISSN: 1311-8080 (printed version); ISSN: 1314-3395 (on-line version)

url: http://www.ijpam.eu

doi: http://dx.doi.org/10.12732/ijpam.v89i1.4

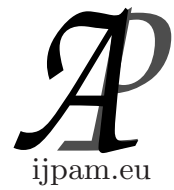

\title{
IMPROVED BOUNDS ON POISSON APPROXIMATION FOR INDEPENDENT BERNOULLI RANDOM SUMMANDS
}

\author{
K. Teerapabolarn \\ Department of Mathematics \\ Faculty of Science \\ Burapha University \\ Chonburi, 20131, THAILAND
}

\begin{abstract}
Bounds for the distance between the distribution function of random sums of independent Bernoulli random variables and an appropriate Poisson distribution function are improved. The bounds in this study are more appropriate for measuring the accuracy of Poisson approximation.
\end{abstract}

AMS Subject Classification: 62E17, 60F05, 60G05

Key Words: Bernoulli random variable, distribution function, Poisson approximation, random sums

\section{Introduction}

Let $X_{1}, X_{2}, \ldots$ be a sequence of independent Bernoulli random variables, each with probability of success $p_{i}=P\left(X_{i}=1\right)=1-P\left(X_{i}=0\right)$, and let $N$ be a nonnegative integer-valued random variable and independent of the $X_{i}$ 's. Let us consider the sum $S_{N}=\sum_{i=1}^{N} X_{i}$, which is called random sums. For the special case, $N=n \in \mathbb{N}$ is fixed, there has been some research on topics related to the approximation of the distribution of the sum $S_{n}$ by an appropriate Poisson distribution, which can be found in [1], [3], [4]-[7], etc. Let $\lambda_{N}=\sum_{i=1}^{N} p_{i}$, $\lambda=E\left(\lambda_{N}\right)$ and $U_{\lambda}$ a Poisson random variable with mean $\lambda$. Yannaros [8] gave

Received: May 16, 2013

(c) 2013 Academic Publications, Ltd. url: www.acadpubl.eu 
a uniform bound for the distance between the distribution functions of $S_{N}$ and $U_{\lambda}$ as follows:

$$
\sup _{x \in \mathbb{N}}\left|P\left(S_{N} \leq x\right)-P\left(U_{\lambda} \leq x\right)\right| \leq E\left|\lambda_{N}-\lambda\right|+E\left(\frac{1-e^{-\lambda_{N}}}{\lambda_{N}} \sum_{i=1}^{N} p_{i}^{2}\right),
$$

and Kongudomthrap and Chaidee [2] gave a non-uniform bound in the form of

$$
\left|P\left(S_{N} \leq x\right)-P\left(U_{\lambda} \leq x\right)\right| \leq \frac{3 \lambda}{x}+E\left\{\frac{1-e^{-\lambda_{N}}}{\lambda_{N}} \min \left\{1, \frac{e^{\lambda_{N}}}{x+1}\right\} \sum_{i=1}^{N} p_{i}^{2}\right\}
$$

where $x \in \mathbb{N}$ and $P\left(S_{N}=0\right)=E\left[\prod_{i=1}^{N}\left(1-p_{i}\right)\right]$.

In this study, we focus on improving the bounds in (1.1) and (1.2) to be more appropriate, which are in Section 2. Concluding remarks are presented in the last section.

\section{Results}

The following theorem presents non-uniform and uniform bounds for the distance between the distribution functions of $S_{N}$ and $U_{\lambda}$, which are the desired results.

Theorem 2.1. For $x \in \mathbb{N}$, then we have

$$
\begin{aligned}
& \left|P\left(S_{N} \leq x\right)-P\left(U_{\lambda} \leq x\right)\right| \leq \min \left\{\frac{2 \lambda}{x+1}, \min \left\{1, \sqrt{\frac{2}{e \lambda}}\right\} E\left|\lambda_{N}-\lambda\right|\right\} \\
& +\min \left\{E\left(\frac{1-e^{-\lambda_{N}}}{\lambda_{N}} \sum_{i=1}^{N} p_{i}^{2}\right), E\left(\frac{2\left(e^{\lambda_{N}}-\lambda_{N}-1\right)}{\lambda_{N}^{2}(x+1)} \sum_{i=1}^{N} p_{i}^{2}\right), \frac{1}{x} E\left(\sum_{i=1}^{N} p_{i}^{2}\right)\right\}
\end{aligned}
$$

and

$$
\begin{gathered}
\sup _{x \in \mathbb{N}}\left|P\left(S_{N} \leq x\right)-P\left(U_{\lambda} \leq x\right)\right| \leq \min \left\{\lambda, \min \left\{1, \sqrt{\frac{2}{e \lambda}}\right\} E\left|\lambda_{N}-\lambda\right|\right\} \\
+\min \left\{E\left(\frac{1-e^{-\lambda_{N}}}{\lambda_{N}} \sum_{i=1}^{N} p_{i}^{2}\right), E\left(\frac{e^{\lambda_{N}}-\lambda_{N}-1}{\lambda_{N}^{2}} \sum_{i=1}^{N} p_{i}^{2}\right)\right\} .
\end{gathered}
$$


Proof. Because (2.2) is directly obtained from (2.1), it suffices to show that (2.1) holds. It follows the fact that

$$
\begin{aligned}
\left|P\left(S_{N} \leq x\right)-P\left(U_{\lambda} \leq x\right)\right| \leq & \sum_{n=0}^{\infty} P(N=n)\left|P\left(S_{n} \leq x\right)-P\left(U_{\lambda} \leq x\right)\right| \\
\leq & \sum_{n=0}^{\infty} P(N=n)\left|P\left(S_{n} \leq x\right)-P\left(U_{\lambda_{n}} \leq x\right)\right| \\
& +\left|P\left(U_{\lambda_{N}} \leq x\right)-P\left(U_{\lambda} \leq x\right)\right|
\end{aligned}
$$

Applying the corresponding results in [5] and [1], we obtain

$$
\left|P\left(S_{n} \leq x\right)-P\left(U_{\lambda_{n}} \leq x\right)\right| \leq \min \left(\frac{1-e^{-\lambda_{n}}}{\lambda_{n}}, \frac{2\left(e^{\lambda_{n}}-\lambda_{n}-1\right)}{\lambda_{n}^{2}(x+1)}, \frac{1}{x}\right) \sum_{i=1}^{n} p_{i}^{2}
$$

and

$$
\left|P\left(U_{\lambda_{N}} \leq x\right)-P\left(U_{\lambda} \leq x\right)\right| \leq \min \left\{1, \sqrt{\frac{2}{e \lambda}}\right\} E\left|\lambda_{N}-\lambda\right|,
$$

respectively. In addition, we have that

$$
\begin{aligned}
\left|P\left(U_{\lambda_{N}} \leq x\right)-P\left(U_{\lambda} \leq x\right)\right| & \leq P\left(U_{\lambda} \geq x+1\right)+\sum_{n=0}^{\infty} P(N=n) P\left(U_{\lambda_{n}} \geq x+1\right) \\
& \leq \frac{E\left(U_{\lambda}\right)}{x+1}+\sum_{n=0}^{\infty} P(N=n) \frac{E\left(U_{\lambda_{n}}\right)}{x+1} \\
& =\frac{\lambda+\lambda}{x+1}=\frac{2 \lambda}{x+1} .
\end{aligned}
$$

Taking these bounds into (2.3), hence (2.1) holds.

If $X_{i}$ 's are identically distributed, then the following corollary is an immediately consequence of the Theorem 2.1.

Corollary 2.1. Let $x \in \mathbb{N}$, if $p_{1}=p_{2}=\cdots=p$, then we have the following:

$$
\begin{gathered}
\left|P\left(S_{N} \leq x\right)-P\left(U_{\lambda} \leq x\right)\right| \leq \min \left\{E\left(1-e^{-p N}\right) p, E\left[\frac{2\left(e^{p N}-p N-1\right)}{N(x+1)}\right],\right. \\
\left.\quad \frac{p^{2} E(N)}{x}\right\}+\min \left\{\frac{2 p E(N)}{x+1}, \min \left\{1, \sqrt{\frac{2}{e E(N) p}}\right\} p E|N-E(N)|\right\}
\end{gathered}
$$


and

$$
\begin{aligned}
\sup _{x \in \mathbb{N}} \mid P\left(S_{N}\right. & \leq x)-P\left(U_{\lambda} \leq x\right) \mid \leq \min \left\{E\left(1-e^{-p N}\right) p, E\left(\frac{e^{p N}-p N-1}{N}\right)\right\} \\
& +\min \left\{p E(N), \min \left\{1, \sqrt{\frac{2}{e E(N) p}}\right\} p E|N-E(N)|\right\} .
\end{aligned}
$$

Remark. Let us consider:

1. $\frac{2 \lambda}{x+1}<\frac{3 \lambda}{x}$

2. $\min \left(\frac{1-e^{-\lambda_{N}}}{\lambda_{N}}, \frac{2\left(e^{\lambda_{N}}-\lambda_{N}-1\right)}{\lambda_{N}^{2}(x+1)}, \frac{1}{x}\right) \leq \frac{1-e^{-\lambda_{N}}}{\lambda_{N}} \min \left\{1, \frac{e^{\lambda_{N}}}{x+1}\right\}$,

3. $\min \left\{\lambda, \min \left\{1, \sqrt{\frac{2}{e \lambda}}\right\} E\left|\lambda_{N}-\lambda\right|\right\} \leq E\left|\lambda_{N}-\lambda\right|$ and

4. $\min \left\{E\left(\frac{1-e^{-\lambda_{N}}}{\lambda_{N}}\right), E\left(\frac{e^{\lambda_{N}}-\lambda_{N}-1}{\lambda_{N}^{2}}\right)\right\} \leq E\left(\frac{1-e^{-\lambda_{N}}}{\lambda_{N}}\right)$.

Thus, the bounds in (2.2) and (2.1) are sharper than the bounds in (1.1) and (1.2) respectively.

\section{Conclusion}

In this study, non-uniform and uniform bounds for the distance between the distribution function of random sums of independent Bernoulli random variables and an appropriate Poisson distribution function were improved. They are sharper than those reported in [8] and [2]. Thus, the bounds obtained in this study are more appropriate for measuring the accuracy of the approximation.

\section{References}

[1] A.D. Barbour, L. Holst, S. Janson, Poisson approximation, Oxford Studies in Probability 2, Clarendon Press, Oxford, 1992.

[2] S. Kongudomthrap, N. Chaidee, Bounds in Poisson approximation of random sums of Bernoulli random variables, Journal of Mathematics Research, 4 (2012), 29-35.

[3] K. Neammanee, Pointwise Approximation of Poisson binomial by Poisson distribution. Stochastic Modelling and Applications, 6 (2003), 20-26.

[4] C.M. Stein, Approximate Computation of Expectations, IMS, Hayward California, 1986. 
[5] K. Teerapabolarn, An improvement of Poisson approximation for sums of dependent Bernoulli random variables, (To be appeared in Communications in Statistics-Theory and Methods.

[6] K. Teerapabolarn, K. Neammanee, Poisson approximation for sums of dependent Bernoulli random variables. ActaMathematica Academiae Paedagogicae Nyiregyhaziensis, 22 (2006), 87-99.

[7] K. Teerapabolarn, T. Santiwipanont, Two non-uniform bounds in the Poisson approximation of sums of dependent indicators, Thai Journal of Mathematics, 5 (2007), 15-39.

[8] N. Yannaros, Poisson approximation for random sums of Bernoulli random variables, Statist. Probab. Lett., 11 (1991), 161-165. 
